\title{
MicroRNA profiling of low-grade glial and glioneuronal tumors shows an independent role for cluster 14q32.31 member miR-487b
}

\author{
Heather Marion Ames ${ }^{1,4}$, Ming Yuan ${ }^{1,4}$, Maria Adelita Vizcaíno ${ }^{1,3}$, Wayne $\mathrm{Yu}^{2}$ and \\ Fausto J Rodriguez ${ }^{1,2}$ \\ ${ }^{1}$ Department of Pathology, Johns Hopkins University School of Medicine, Baltimore, MD, USA; ${ }^{2}$ Sidney \\ Kimmel Comprehensive Cancer Center, Johns Hopkins University School of Medicine, Baltimore, MD, USA \\ and ${ }^{3}$ Department of Cellular and Tissue Biology, Universidad Nacional Autónoma de México, Mexico City, \\ $D F, U S A$
}

\begin{abstract}
Low-grade (WHO I-II) gliomas and glioneuronal tumors represent the most frequent primary tumors of the central nervous system in children. They often have a good prognosis following total resection, however they can create many neurological complications due to mass effect, and may be difficult to resect depending on anatomic location. MicroRNAs have been identified as molecular regulators of protein expression/translation that can repress multiple mRNAs concurrently through base pairing, and have an important role in cancer, including brain tumors. Using the NanoString digital counting system, we analyzed the expression levels of 800 microRNAs in nine low-grade glial and glioneuronal tumor types $(n=45)$. A set of 61 of these microRNAs were differentially expressed in tumors compared with the brain, and several showed levels varying by tumor type. The expression differences were more accentuated in subependymal giant cell astrocytoma, compared with other groups, and demonstrated the highest degree of microRNA repression validated by RT-PCR, including miR-129-2-3p, miR-219-5p, miR-338-3p, miR-487b, miR-885-5p, and miR-323a-3p. Conversely, miR-4488 and miR-1246 were overexpressed in dysembryoplastic neuroepithelial tumors compared with the brain and other tumors. The cluster 14q32.31 member miR-487b was variably under-expressed in pediatric glioma lines compared with human neural stem cells. Overexpression of miR-487b in a pediatric glioma cell line (KNS42) using lentiviral vectors led to a decrease in colony formation in soft agar $(30 \%)(P<0.05)$, and decreased expression of known predicted targets PROM1 and Nestin (but not WNT5A). miR-487b overexpression had no significant effect on cell growth, proliferation, sensitivity to temozolomide, migration, or invasion. In summary, microRNA regulation appears to have a role in the biology of glial and glioneuronal tumor subtypes, a finding that deserves further investigation.

Modern Pathology (2017) 30, 204-216; doi:10.1038/modpathol.2016.177; published online 14 October 2016
\end{abstract}

The category of low-grade gliomas is composed of a diverse group of slow growing tumors that primarily arise in childhood and young adulthood. The related category of glioneuronal tumors demonstrate similar demographics, although they are more heterogeneous with regard to specific subtypes, and can have a variable proportion of glial and neuronal components. Both of these tumor types are initially

Correspondence: Dr FJ Rodriguez, MD, Division of Neuropathology, Johns Hopkins Hospital, Sheikh Zayed Tower, Room M2101, 1800 Orleans Street, Baltimore, MD 21231, USA.

E-mail: frodrig4@jhmi.edu

${ }^{4}$ Co-first authors.

Received 22 May 2016; revised 23 August 2016; accepted 24 August 2016; published online 14 October 2016 treated by surgery and are associated with a good prognosis, although they may be associated with seizures and deleterious mass effects. Association with genetic syndromes such as neurofibromatosis and tuberous sclerosis may be present and malignant transformation is very rare. With these characteristics, low-grade gliomas and glioneuronal tumors represent a diverse, yet genetically stable, pool from which to identify potential molecular targets.

Our understanding of the biology of pediatric lowgrade glioma has benefited from major recent advances in molecular genetics. The application of high resolution platforms has discovered alterations not recognizable through traditional cytogenetic analysis. For example, BRAF-KIAA1549 fusions are 
the most frequent recurrent alteration in pilocytic astrocytoma, ${ }^{1-5}$ the predominant subtype of pediatric low-grade astrocytoma. BRAF-KIAA1549 fusions, as well as other genetic rearrangements and mutations lead to downstream activation of signaling pathways, particularly the mitogenactivated protein kinase pathway. ${ }^{2}$ More recently, comprehensive sequencing studies have documented genetic hits in mitogen-activated protein kinase pathway components in essentially $100 \%$ of pilocytic astrocytomas. ${ }^{6}$ In patients with neurofibromatosis type 1, pilocytic astrocytomas develop homozygous mutations in the NF1 gene, also leading to MAPK pathway activation. Another relevant signaling pathway, involving the mammalian target of rapamycin (mTOR), is frequently activated in pediatric lowgrade glioma, ${ }^{7,8}$ and represents the key molecular property of subependymal giant cell astrocytoma, a tumor frequently developing in the setting of tuberous sclerosis, and characterized by inactivation of TSC1 or TSC2. Other pediatric low-grade glioma subsets have different alterations, for example, partial duplication of the transcription factor MYBL1 with truncated transcript, intragenic duplications of the tyrosine kinase domain in the FGFR1 gene, and $M Y B$ rearrangements in diffuse pediatric low-grade gliomas. $^{9,10}$

A role for a variety of noncoding ribonucleic acid molecules (RNAs), particularly microRNAs (measuring $~ 22$ nucleotides in length), has been increasingly documented in many normal and abnormal physiologic states, including cancer. MicroRNAs have been identified as regulators of RNA transcription and protein translation. Through this mechanism, multiple mRNAs can be concurrently targeted through base pairing. Tumor suppressors may be targeted through microRNA upregulation, whereas oncogenes may be increased in abundance by downregulation of corresponding microRNAs. Of relevance to this study, several microRNAs have been implicated in gliomagenesis by prior studies (eg, miR-21, miR-7, miR-181a/b, miR-221, and miR-22211-15), and also regulate signaling pathways in diffuse gliomas, including glioblastoma. ${ }^{16,17}$ For example, PTEN, a validated tumor suppressor gene, is frequently inactivated in diffuse gliomas, and may be regulated by a number of microRNAs such as miR-21, miR-23a, and miR-26a resulting in increased $\mathrm{AKT} / \mathrm{mTOR}$ activity. ${ }^{18,19}$ MicroRNAs may have more than one target, and therefore microRNA profiling may uncover molecular subgroups of cancer more robustly than conventional mRNA profiling. ${ }^{20}$ Lowgrade pediatric gliomas lack the multiple overt genomic alterations typical of higher grade tumors, ${ }^{21}$ and therefore microRNA and other genetic/epigenetic mechanisms are likely to participate in their pathogenesis. Our work and that of others has described differentially expressed microRNAs in pilocytic astrocytomas compared with normal brain, including miR-21, miR-124, and miR-129. ${ }^{2-25}$ Some studies have also reported differential microRNA expression in other pediatric brain tumors, such as medulloblastoma and ependymoma. ${ }^{26}$ Studies of microRNA regulation in other low-grade glioma subtypes and glioneuronal tumors are lacking, with some inflammationassociated microRNAs affected in ganglioglioma. ${ }^{27,28}$ In the current study, we investigated global microRNA expression in a series of low-grade glioma and glioneuronal subtypes with the aim of identifying biologic and clinically relevant targets.

\section{Materials and methods}

\section{Tumor Samples}

Cases of low-grade glioma or glioneuronal tumors were queried in the pathology database of Johns Hopkins Hospital. All available slides were reviewed by two of the authors (FJR, MAV). Only sections with $>60 \%$ neoplastic cellularity were selected for further study. Formalin-fixed paraffin-embedded sample rolls were collected in eppendorf tubes. RNA extractions and quality analysis were performed at the Sidney Kimmel Comprehensive Cancer Center Microarray Core Facility at the Johns Hopkins University (Baltimore, MD, USA) using the miRNeasy formalin-fixed paraffin-embedded tissue Kit (Qiagen). A total of 45 samples were included in the study representing all categories of low-grade, primarily pediatric, gliomas and glioneuronal tumors. Mean age was 15 years (range: 17 weeks to 57 years). Diffuse gliomas in adults and NF1-associated tumors were excluded. Demographic features of these tumors per case are outlined in Supplementary Table 1. Tumor samples included subependymal giant cell astrocytoma $(n=6)$, pilocytic astrocytoma $(n=6)$, pleomorphic xanthoastrocytomas $(n=7)$, gangliogliomas $(n=6)$, dysembryoplastic neuroepithelial tumor $(n=5)$, angiocentric glioma $(n=3)$, pediatric oligodendroglioma $(n=3)$, pediatric diffuse astrocytoma $(n=3)$, rosette-forming glioneuronal tumor $(n=2)$, and non-neoplastic brain controls $(n=4)$. All studies were performed using established ethical guidelines and institutional review board approval.

\section{MicroRNA Profiling}

NanoString technology was applied to formalinfixed paraffin-embedded tissues to quantify the global expression of 800 microRNAs in nine types of low-grade neoplasms of the brain. Raw data was normalized using nSolver software (NanoString), filtered for minimum expression threshold, and $\mathrm{log}_{2}$ transformed before analysis. $\mathrm{MeV}$ software (version 4.8.0) was used to generate unsupervised hierarchical clusters following significance evaluation via significant analysis of microarray. Results for 10 microRNAs of interest were further validated using standard Taq-man RT-PCR protocols and 
probes (Life Technologies). The demographics of the samples used for RT-PCR validation are indicated by a star $\left(^{*}\right)$ in Supplementary Table 1.

\section{Cell Culture}

Pediatric glioma-derived lines Res186, Res259, UW479, and SF-188 have been previously described, ${ }^{29}$ and were kindly provided by Dr. Chris Jones (Institute of Cancer Research, Sutton, UK). CHLA-200 and SJ-GBM2 were provided by the Children's Oncology Group Cell Culture and Xenograft Repository banks. The pediatric glioma cell line KNS42 was obtained from the Japan Cancer Research Resources cell bank. Details of the various cell lines is summarized in Supplementary Table 2. Cells were cultured in Dulbecco modified Eagle medium/ F12 Ham medium (DMEM/F12) supplemented with 10\% heat-inactivated fetal bovine serum. TrypLE express enzyme was used for cell dissociation. Nonneoplastic human neural stem cells have been previously described ${ }^{30}$ and were grown in DMEM/ F12 supplemented with 20\% B27 supplement, $5 \mu \mathrm{g} /$ $\mathrm{ml}$ heparin, $20 \mathrm{ng} / \mathrm{ml} \mathrm{EGF}$, and $20 \mathrm{ng} / \mathrm{ml}$ FGF2. All culturing reagents were purchased from ThermoFisher Scientific.

\section{miRNA Overexpression and Inhibition}

Lentiviral-based miRNA plasmids were obtained from Biosettia, including expression plasmids $\mathrm{pLV}$ hsa-mir-487b (mir-487b) and pLV-mir-control (mircontrol), inhibition plasmids pLV-hsa-mir-1246 locker (mir-1246 locker), and pLV-mir-locker control (mir-locker). To produce lentiviruses, 293T cells were transfected with miRNA plasmid and VSVG packaging plasmids mixture using Lipofectamine 2000 (ThermoFisher Scientific). Lentiviral supernatants were collected at $72 \mathrm{~h}$ post transfection, and concentrated using Polyethylene glycol (PEG) 8000. Virus was resuspended in DMEM and kept frozen at $-80^{\circ} \mathrm{C}$ until needed. KNS42 cells were infected with virus in the presence of polybrene ( $5 \mu \mathrm{g} / \mathrm{ml}$, Sigma-Aldrich). At $48 \mathrm{~h}$ later, infected cells were selected with $1 \mu \mathrm{g} / \mathrm{ml}$ of puromycin (SigmaAldrich, for expression plasmids) or $5 \mu \mathrm{g} / \mathrm{ml}$ of Blastidin (ThermoFisher Scientific, for inhibition plasmids) for 5 days to generate stable cell lines. Target prediction was performed using TargetScan, version $6.2 .{ }^{31}$

\section{Cell Cycle Analysis}

Cells were dissociated and fixed with $70 \%$ Ethanol at $4{ }^{\circ} \mathrm{C}$ overnight, then stained with Muse cell cycle reagent for $30 \mathrm{~min}$ at room temperature and analyzed with Muse flow cytometer (Millipore) according to the manufacturer's instructions. Cell cycle data were analyzed with FlowJo software.

\section{Cell Growth and Cell Survival Assay}

The CellTiter-Blue cell viability assay kit (Promega) was used to count viable cells. Cells were seeded in 96 well plates at a density of 1000 cells per well in growth media. Twenty microliters of CellTiter-Blue reagent was added to $100 \mu \mathrm{l}$ medium and incubated for $1 \mathrm{~h}$ at $37^{\circ} \mathrm{C}$ incubator. Fluorescence (560 nm Ex/ $590 \mathrm{~nm}$ Em) was measured using TECAN plate reader. For cell growth assay, relative cell numbers were continuing measured for 5 days. For drug treatment, cells were cultured in the media with different doses of temozolomide (TMZ, Skellchem) for 7 days, DMSO was used as control.

\section{Quantitative Real-Time Polymerase Chain Reaction (qRT-PCR)}

Primers for miRNA were designed by using miRprimer software. $^{32}$ Sequences can be found in Supplementary Table 3, and small RNA RNU48 was used as the endogenous control. Primer sequences for mRNA target genes are listed in Supplementary Table 4, and HPRT1 was used as the endogenous control. Total RNA was isolated from cultured cells using miRNeasy mini kit (Qiagen), reverse transcription of miRNA was performed per published protocols ${ }^{32}$ and cDNA for target genes were produced using QuantiTect reverse transcription kit (Qiagen). qRT-PCR was performed using SsoAdvanced universal SYBR green supermix (BioRad). The relative fold change was calculated based on the difference of Ct values.

\section{In Vitro Invasion Assay}

Cell invasion assay was analyzed using a 24 well system with growth factor reduced Matrigel coated transwell inserts containing $8 \mu \mathrm{M}$ pore membrane (Corning). KNS42 cells $\left(1 \times 10^{5}\right)$ in $250 \mu \mathrm{l}$ of DMEM/ F12 were added on top of each membrane and fill bottom with $750 \mu \mathrm{l}$ of complete growth medium. After $24 \mathrm{~h}$, migrated cells were dissociated from the membrane by using Accutase (Sigma-Aldrich) and resuspended in growth medium. Relative cell numbers were measured by using CellTiter-Blue reagent (Promega).

\section{Wound Healing Assay}

Cells were grown in 12-well plates to confluence, then scratched with 1 P1000 pipette tip. The cells were rinsed with PBS, imaged, and allowed to migrate for $48 \mathrm{~h}$ before taking a second image. ImageJ software (National Institutes of Health) was used to quantify cell migration. 


\section{Soft Agar Clonogenic Assay}

One thousand cells were resuspended in growth medium with $0.4 \%$ soft agar, then seeded in 6-well plate with $0.8 \%$ bottom agar layer. After 21 days, cells were fixed and stained with $0.01 \%$ Crystal violet in $20 \%$ methanol. The number of colonies containing more than 50 cells in each well were counted using a Nikon inverted microscope.

\section{Statistical Analysis of Cell Culture Assays}

All data are presented as mean \pm s.d., and $P<0.05$ was considered significant. All experiments were performed in at least three biological replicates and data were analyzed with a two-tailed Student's $t$-test.

\section{Results}

MicroRNA Profiling Reveals Differentially Expressed MicroRNAs in Low-Grade Glioma and Glioneuronal Tumors Compared with Brain

In an unsupervised hierarchical analysis of microRNA expression data, non-neoplastic brain samples clustered together, whereas subependymal giant cell astrocytomas and rosette-forming glioneuronal tumors segregated furthest from non-neoplastic brain (Figure 1a). A total of 61 microRNAs showed altered expression among the 10 groups of samples as evaluated by significance analysis of microarrays.
These microRNAs included a similar list of those that have been previously identified as having altered expression in pilocytic astrocytomas as compared with normal brain. Specifically, miR129-5p, which was previously validated as downregulated in pilocytic astrocytomas, was also downregulated to a varying extent in all low-grade gliomas and glioneuronal tumors evaluated in this study. Both subependymal giant cell astrocytomas and pilocytic astrocytomas had significant decreases in miR129-5p expression $(P<0.05)$ (Figure 1b), leading us to hypothesize that subependymal giant cell astrocytomas may also have increased staining for the nuclear proteins PBX3 and NFI/B, as previously described in pilocytic astrocytoma. ${ }^{23}$ Subependymal giant cell astrocytomas $(n=3)$ were stained for PBX3 and NFI/B according to previously established protocols ${ }^{23}$ and were scored for nuclear staining on a 0-3 scale as compared with normal brain. As expected, subependymal giant cell astrocytomas showed strong $(3+)$ nuclear staining for PBX3 (2 of 3 ) and NFIB (3 of 3 ) (Figure 1c).

\section{Coordinated Repression of Cluster 14q32.31}

MicroRNAs in Low-Grade Gliomas and Subependymal Giant Cell Astrocytomas as Compared with Low-Grade Glioneuronal Tumors

When looking at global microRNA expression, subependymal giant cell astrocytomas, pilocytic

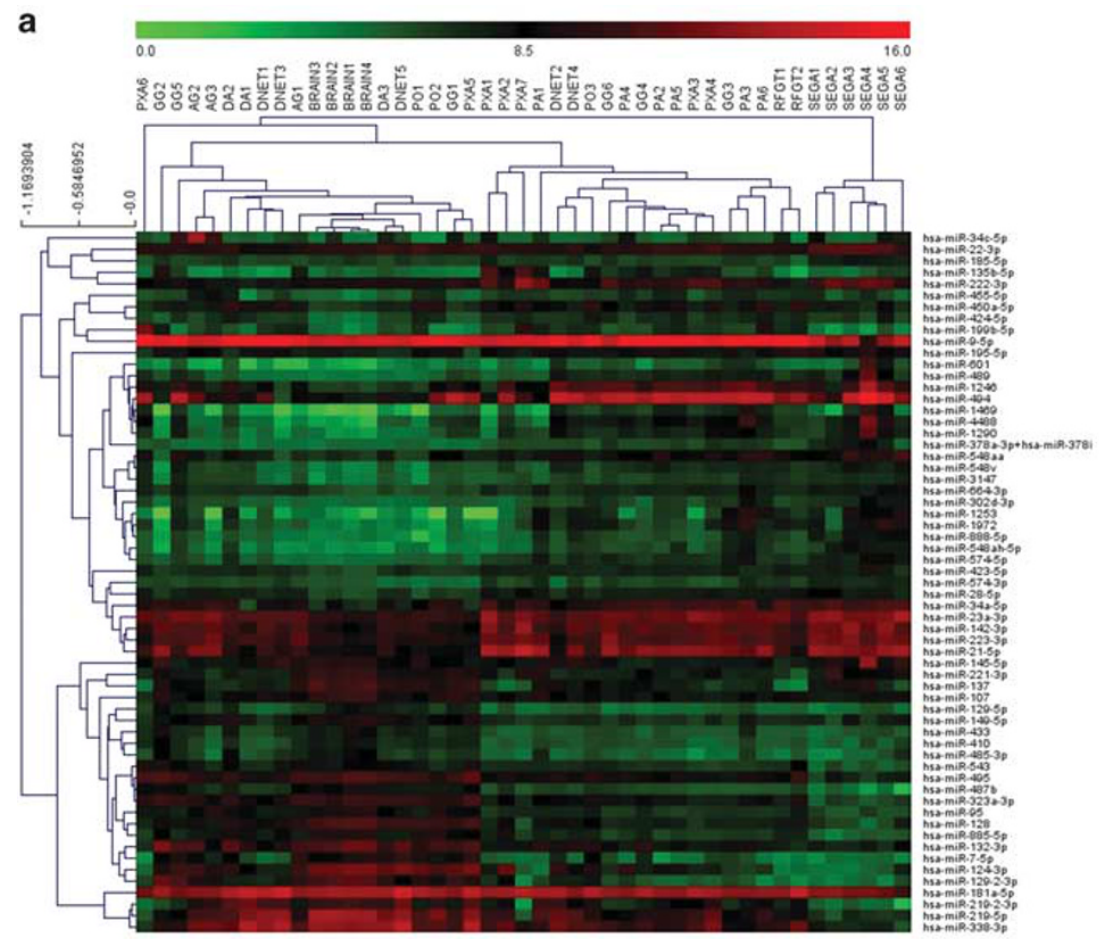

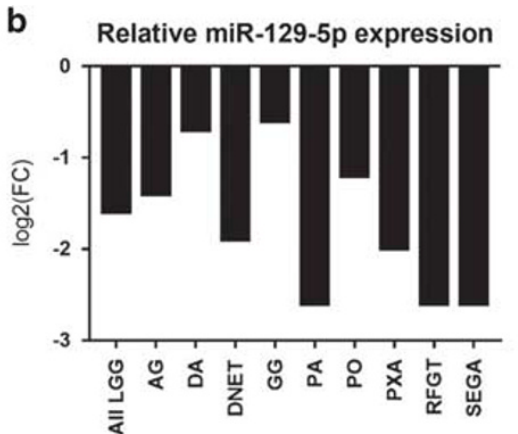
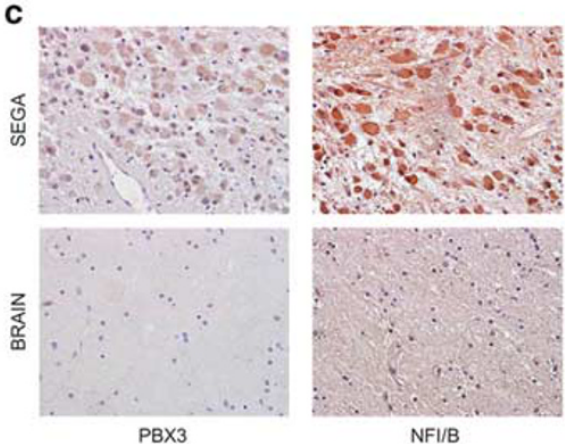

Figure 1 Hierarchical clustering of miRNA expression levels in low-grade gliomas and glioneuronal tumors. (a) MeV generated heatmap of microRNAs with significantly altered expression among the 10 subgroups as evaluated by significance analysis of microarrays. (b) Log 2 (fold change) of mean normalized expression values for miR129-5p as compared with brain $\left({ }^{*} P<0.05\right)$. (c) Immunohistochemical staining for miR-129-5p targets PBX3 and NFIB in normal brain and SEGAs. 
astrocytomas, and rosette-forming glioneuronal tumors clustered together and farthest from nonneoplastic brain, whereas dysembryoplastic neuroepithelial tumor, ganglioglioma, and pleomorphic xanthoastrocytoma segregated closer to normal brain (Figure 1a). This raised the possibility that as dysembryoplastic neuroepithelial tumor, ganglioglioma, and pleomorphic xanthoastrocytoma have a mixed cellular composition, the heterogeneous elements may lead to relative clustering with brain compared with the homogeneous, well-circumscibed subependymal giant cell astrocytomas, and pilocytic astrocytomas. To further identify those microRNAs that are differentially expressed in homogenous glial tumors (subependymal giant cell astrocytomas and pilocytic astrocytomas) and heterogeneous glioneuronal tumors (gangliogliomas and dysembryoplastic neuroepithelial tumors), significance analysis of microarrays was performed to directly compare these two groups. This yielded a list of 35 microRNAs that were differentially expressed between the two groups, of which all but five were identified as significant on the initial analysis of all 10 groups (Figure 2a). In addition, there was a similar directionality for both glial and glioneuronal tumors vs brain for the 30 microRNAs that were both significant among all 10 groups and between the wellcircumscribed glial and glioneuronal tumors, with the greatest fold changes seen in subependymal giant cell astrocytomas (Figure 2b).

Given the enrichment in subependymal giant cell astrocytomas of significantly altered microRNAs in the low-grade gliomas, significance analysis of microarrays was performed with unsupervised hierarchical clustering of differentially regulated microRNAs compared between subependymal giant cell astrocytomas and brain. This yielded 67 microRNAs with significantly altered expression, with 42 microRNAs upregulated in subependymal giant cell astrocytomas (Figure 3a) and 25 microRNAs downregulated (Figure 3b). It was notable that within the negatively regulated genes there were multiple a

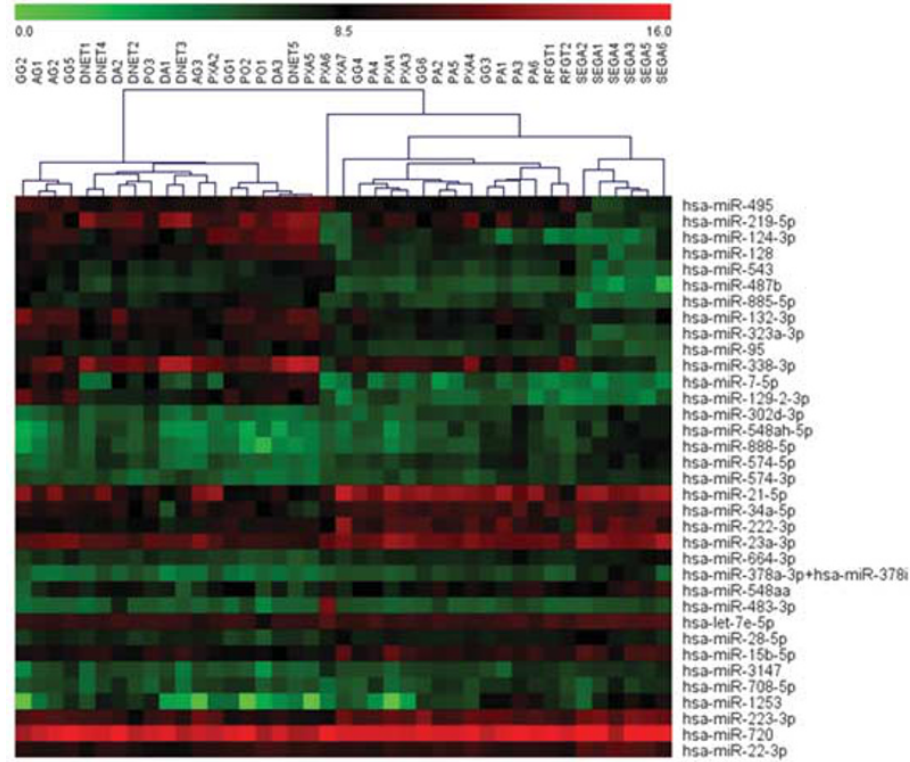

b

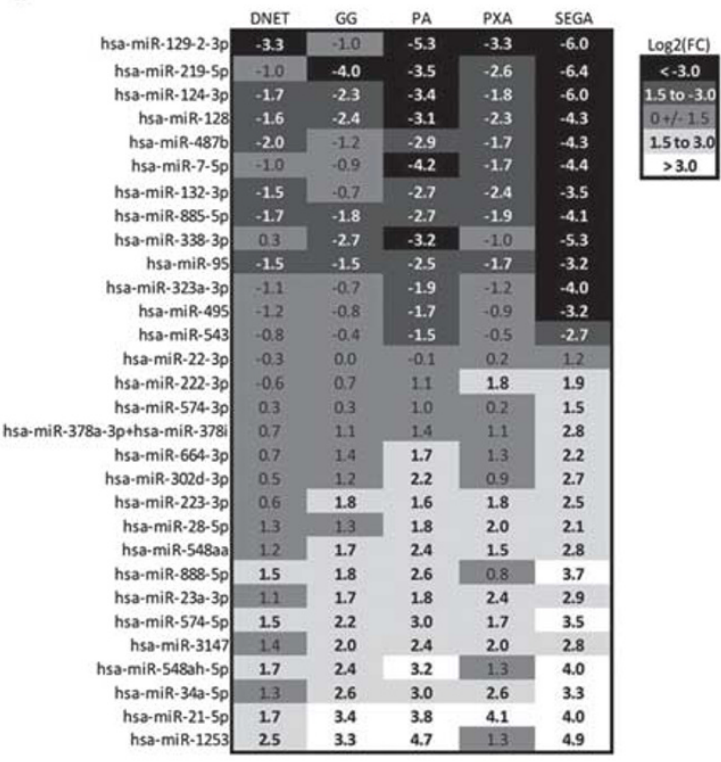

Figure 2 Differentially expressed microRNAs in well-circumscribed low-grade gliomas compared with glioneuronal tumors. (a) Heatmap showing a subset of microRNAs that are differentially expressed in well-circumscribed low-grade gliomas (subependymal giant cell astrocytomas and pilocytoic astrocytomas) as compared with low-grade glioneuronal tumors (ganglioglioma and dysembryoplastic neuroepithelial tumor). (b) Mean $\log _{2}$ (fold change) from normal brain for the tumor types with $n>3$ in our data set (subependymal giant cell astrocytoma, pilocytoic astrocytoma, pleomorphic xanthoastrocytoma, ganglioglioma, and dysembryoplastic neuroepithelial tumor) shows similar directionality in expression among all groups.

Figure 3 Coordinated repression of cluster 14q32.31 microRNAs in low-grade gliomas. (a) Heatmap showing a hierarchical cluster of microRNAs that are upregulated in subependymal giant cell astrocytoma as compared with normal brain. (b) Heatmap showing a hierarchical cluster of microRNAs that are downregulated in subependymal giant cell astrocytoma as compared with normal brain that contains several members of the 14q32.31 cluster of microRNAs (green bar). (c) Mean $\log _{2}$ (fold change) from normal brain for the tumor types with $I>3$ in our data set (subependymal giant cell astrocytoma, pilocytoic astrocytoma, pleomorphic xanthoastrocytoma, ganglioglioma, and dysembryoplastic neuroepithelial tumor) shows similar directionality in expression among all groups for 14q32.31 cluster microRNAs. (d) Heatmap showing a hierarchical cluster of microRNAs that have significantly different expression between SEGA and PA. 
microRNAs in the neurodevelopmentally regulated $14 q 32.31$ cluster that were significantly downregulated within a single hierarchical cluster (Figure 3b).
Looking at the $\log _{2}$ (fold change) of every $14 q 32.31$ microRNA that showed sufficient expression for analysis, the majority of these microRNAs were

a
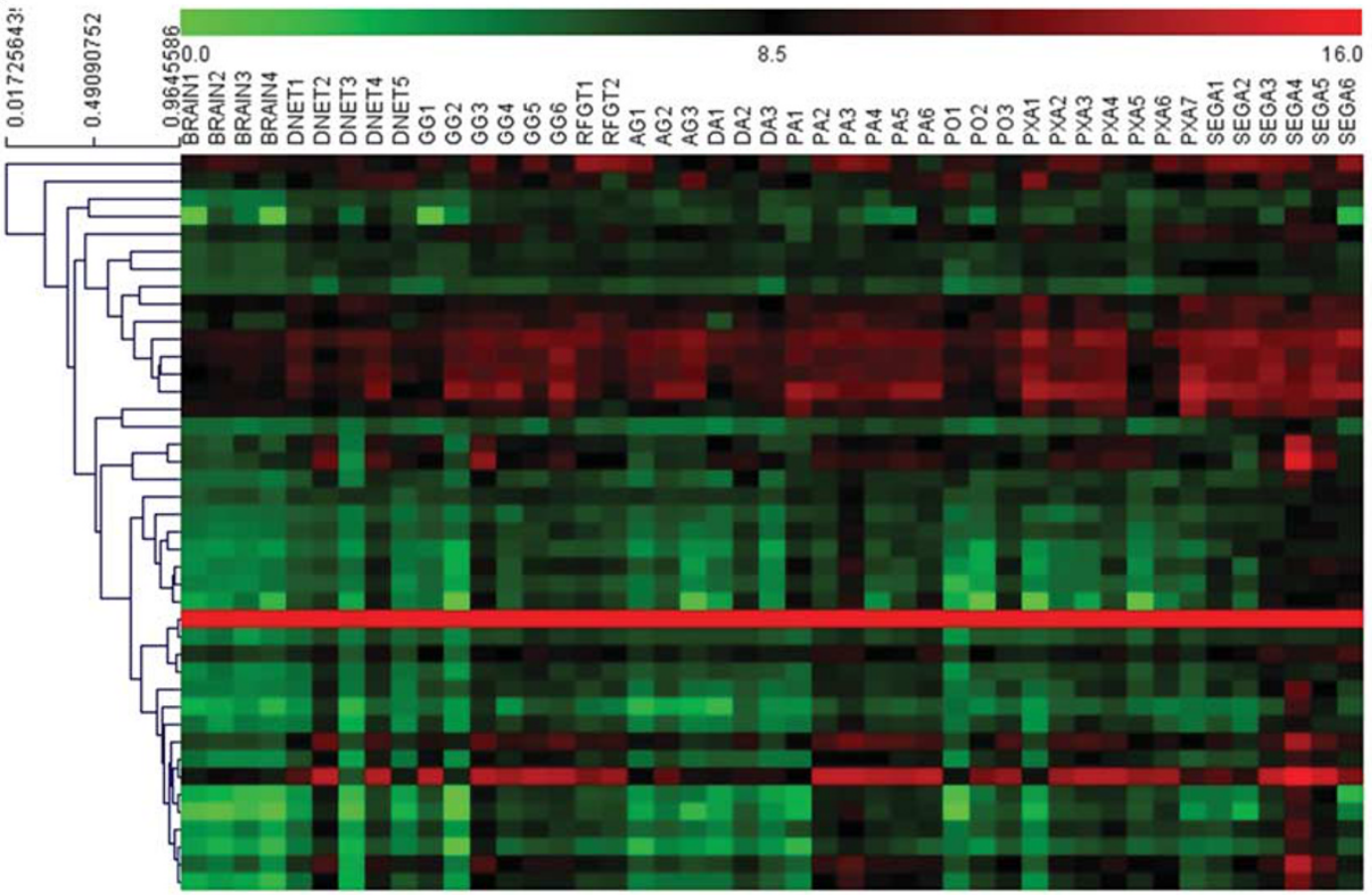

hsa-miR.2045p

hssa-miR.

hsa-miR. 1283

hsa-mir. $143 \mathrm{a}-3 \mathrm{p}$

hsa-miR.28.5p

hsa.miR.-5743p

hsa-miR. $15 b-5 p$
hsa-miR. $342-5 p$

hsa.miR-23a-3p

hsa-miR.223.3p

hsa-miR. $142.3 p$
hss-miR. $21.5 p$

hsa-miR.21.5p

hss. miR.-3783-3p+hss-mir-378

hsa-mir.-575
hsa.miR-630

hsa-miR.320e

hsa-mik. $6043 p$

hsa.miR. $5745 \mathrm{p}$

hsa-miR-548ah-5p

hsa.miR.1972

hsa.miR.
hsa.miR. 1253 .
his

hsa-miR.4454

hsa-miR-3147

hsa-miR.548aa

hsar-miR. $548 \mathrm{~V}$

hsa-miR-e01

hss.miR. 4284

hsa-miR. 1246

hsa-miR.1973

hsa-mik. 404

hsa-miR. 1429

hsa-miR. 1290

hsa-miR.1015-3p

hsalmik. 4516

b

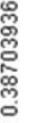

ปิ

สิ

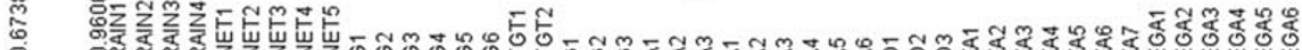
o

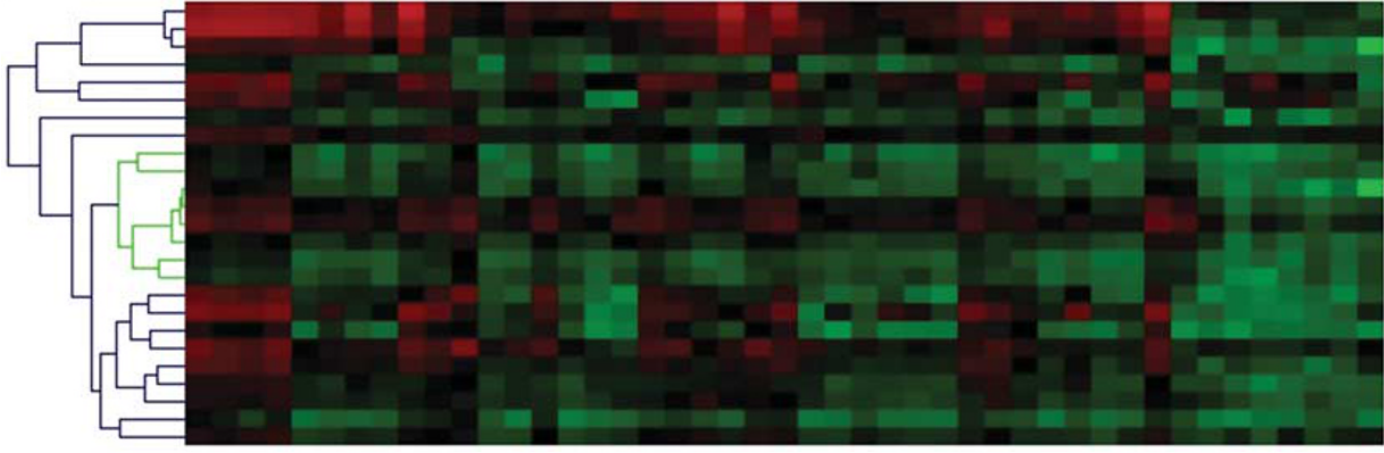

hsa-miR-338-3p hsa-miR.219.5p

hsa-miR.219.2.3p

hsa-miR.218.5p

hsa-miR. 137

hsa-miR-377.3p

hsa-miR.107

hsa-miR.485.-3p

hsa-miR.43
has-miR.437b

hsa-miR-323a-3p

hsa-miR.405

hsa-miR.-54

hsa-mik-410

hsa-miR. 120.2-3p

hss-miR.-1243p

hsa-miR. $7.5 \mathrm{p}$
hsa-miR. $132 \cdot 3 \mathrm{p}$

hsa-miR
hss-miR.128

hsa-miR. 128

hss-miR.-95

hsa-miR.129.5p
hsa-miR. $140-5 p$

hsa-miR-485-3p hsa-miR-487b hsa-miR-410 hsa-miR-323a-3p hsa-mir-323ahsa-mik. 495 hsa-miR-381 hsa-miR-377-3 hsa-miR-543 hsa-miR-656 hsa-miR-487a hsa-miR-376c hsa-miR-379.5p hsa-mik-299.5p hsa-miR. $411.5 p$ hsa-miR-382-5 hsa-miR-376a-3p hsa-miR-299.3 hsa-miR-154-5 hsa-miR-376b hsa-miR-494

\section{d}
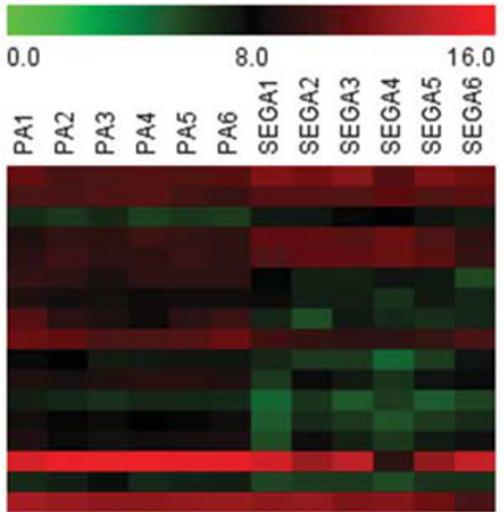

hsa.miR-223-3p hsa.let.7e-5p sa.miR-29c-3p hsa-miR-22-3p hsa-miR-338-3p hsa-miR-181c.5p hsa-miR-219.5p hsa-miR-130a-3p hsa.miR. 128

isa-miR.495

hsa-miR.543

hsa-miR-323a-3p

hsa-mik-376c

hsa-miR.598

hsa-miR-181 a-5p 


\begin{tabular}{|c|c|c|c|c|c|c|c|}
\hline & All LGG & SEGA & PA & PXA & DNET & GG & \\
\hline hsa-miR-129-2-3p & -2.6 & -6.0 & -5.3 & -3.3 & -3.3 & -10 & $\log 2(F C)$ \\
\hline hsa-miR-219-5p & -2.5 & -6.4 & -3.5 & -26 & -1.0 & -4.0 & $<-3.0$ \\
\hline hsa-miR-487b & -1.9 & -4.3 & -2.9 & -1.7 & -2.0 & -1.2 & 1.5 to -3.0 \\
\hline hsa-miR-885-5p & -1.8 & -4.1 & -2.7 & -1.9 & -1.7 & -1.8 & $0+1.1 .5$ \\
\hline hsa-miR-338-3p & -1.2 & -5.3 & -3.2 & -1.0 & 0.3 & -2.7 & 1.5 to 3.0 \\
\hline hsa-miR-323a-3p & -1.2 & -4.0 & -1.9 & -1.2 & -1.1 & -0.7 & $>3.0$ \\
\hline hsa-miR-34a-5p & 2.6 & 3.3 & 3.0 & 2.6 & 1.3 & 2.6 & \\
\hline hsa-miR-21-5p & 3.4 & 4.0 & 3.8 & 4.1 & 1.7 & 3.4 & \\
\hline hsa-miR-1246 & 4.1 & 5.1 & 4.6 & 3.5 & 3.8 & 4.1 & \\
\hline hsa-miR-4488 & 5.4 & 7.0 & 5.9 & 4.5 & 4.1 & 4.6 & \\
\hline
\end{tabular}

b
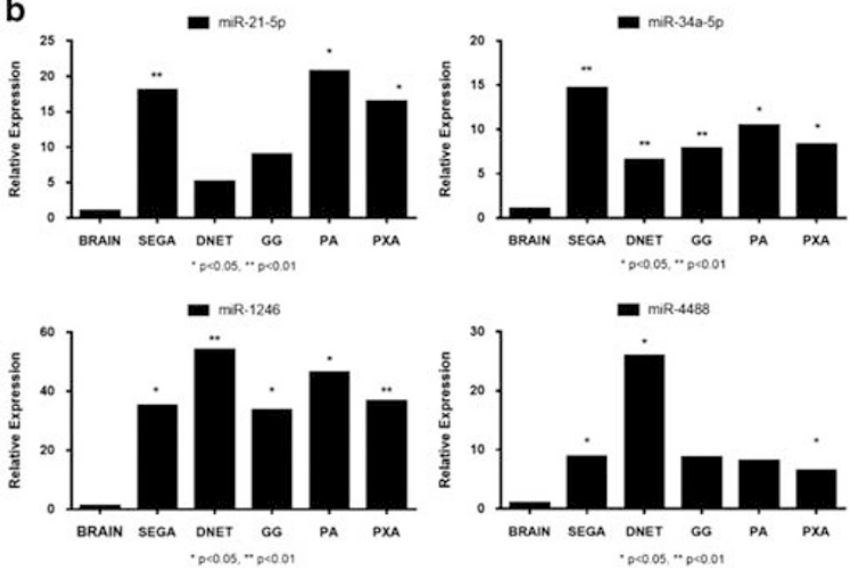
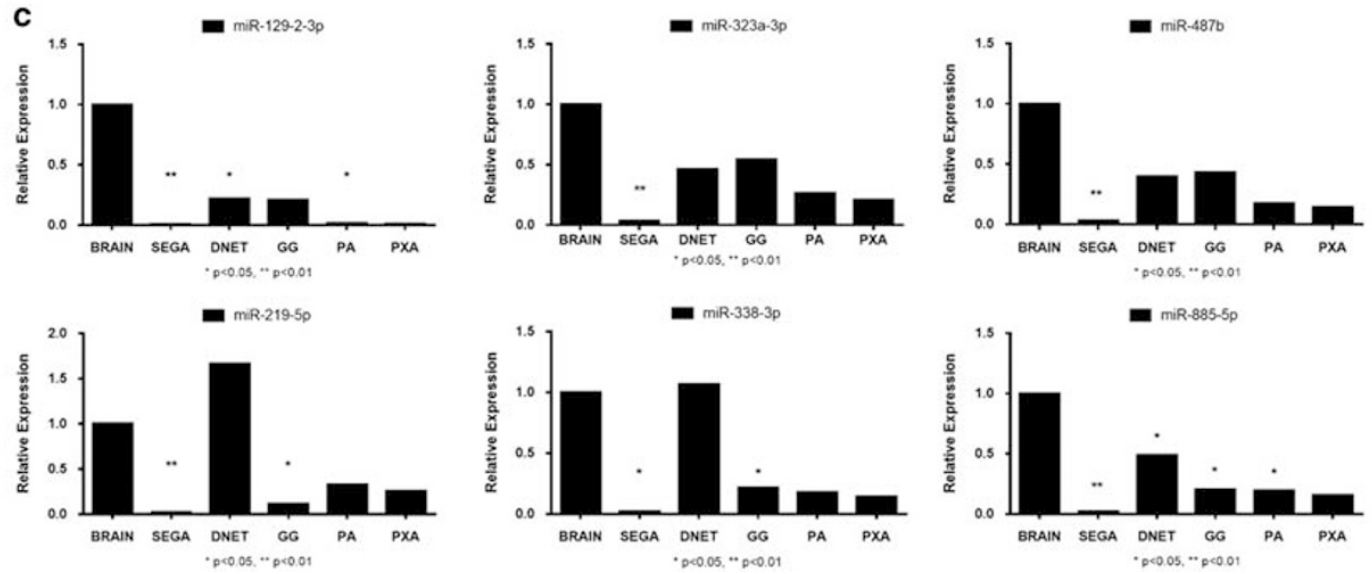

Figure 4 RT-PCR evaluation of differentially expressed microRNAs. (a) Mean $\log _{2}$ (fold change) from normal brain for the tumor types with $n>3$ in our data set (subependymal giant cell astrocytoma, pilocytoic astrocytoma, pleomorphic xanthoastrocytoma, ganglioglioma, and dysembryoplastic neuroepithelial tumor) for miRNAs further evaluated in RT-PCR experiments. (b) RT-PCR results for microRNAs that were upregulated in low-grade glial and glioneuronal tumors. (c) RT-PCR results for microRNAs that were downregulated in lowgrade glial and glioneuronal tumors. ${ }^{*} P<0.05$, ${ }^{*} P<0.01$.

downregulated in each low-grade tumor type (Figure 3c), with the exception of miR-494, which was significantly upregulated in subependymal giant cell astrocytomas and many of the low-grade glial and glioneuronal tumors (Figure 3a), and miR-376b, which had a similar upward trend (Figure 3c).

To determine whether there were significant differences in expression between the wellcircumscribed low-grade gliomas evaluated for altered microRNA expression in our Nanostring analysis (subependymal giant cell astrocytoma and pilocytic astrocytoma), significance analysis of microarrays was performed between these two groups. Interestingly, this yielded only 17 significantly different microRNAs, of which 12 were previously identified as being significantly altered in low-grade gliomas as compared with both brain and glioneuronal tumors in our prior analyses (Figure 3d). None of the remaining five microRNAs had a notably large fold change $\left(-1.5<\log _{2}\right.$ [fold change] $\left.>1.5\right)$ between the two tumors.

For further evaluation by RT-PCR, miR-487b along with five other microRNAs (miR-129-2-3p,
miR-219-5p, miR-338-3p, miR-487b, miR-885-5p, and miR323a-3p) that were significantly downregulated in subependymal giant cell astrocytomas and pilocytic astrocytomas as compared with ganglioglioma and dysembryoplastic neuroepithelial tumor, and two microRNAs (miR-21-5p and miR-34a-3p) that were significantly upregulated in subependymal giant cell astrocytomas and pilocytic astrocytomas were selected. In addition, miR-1246 and miR-4488, which showed global overexpression in the lowgrade tumors, were evaluated. The $\log _{2}$ (fold change) expression of these microRNAs in tumors as compared with normal brain is illustrated in Figure 4a. RT-PCR for these microRNAs was then performed from paraffin-derived RNA from subependymal giant cell astrocytoma, pilocytic astrocytoma, dysembryoplastic neuroepithelial tumor, ganglioglioma, and pleomorphic xanthoastrocytoma.

Of all the tumors studied, subependymal giant cell astrocytomas demonstrated the highest degree of microRNA repression validated by RT-PCR, including miR-129-2-3p, miR-219-5p, miR-338-3p, miR487b, miR-885-5p, and miR-323a-3p (Figure 4a). When looking at the differentially overexpressed 
microRNAs, miR-21, and miR-34a were overexpressed to a greatest extent in subependymal giant cell astrocytoma, followed by pilocytic astrocytoma (Figure 4b). Of interest, miR-4488 and miR-1246 were more highly overexpressed in dysembryoplastic neuroepithelial tumor compared with brain and other tumors $(P<0.05)$, but no repression of miR-219 and miR-338, two microRNAs involved in oligodendrocyte differentiation, was identified.

\section{mir-487b Overexpression Affects Stem Cell Markers and Colony Formation in Soft Agar}

Next, we selected miR-487b (under-expressed in low-grade tumors) and miR-1246 (overexpressed in low-grade tumors) for additional functional experiments. miR-1246 is activated by p53, and suppresses the Down syndrome associated protein (DYRK1a). The Targetscan algorithm predicted miR-487 to directly target several proteins involved in stem cell biology including PROM1, Nestin, and WNT5A. miR-487b and miR-1246 were similarly expressed in pediatric glioma lines as compared with neural stem cells, although miR-1246 was relatively enriched in tumors versus stem cells (Supplementary Figures $1 \mathrm{~A}$ and 2A). Next, miR-487b was overexpressed using a lentiviralbased miRNA plasmid, whereas miR-1246 knockdown was attempted with a pLV-hsa-mir-1246 locker in the same pediatric glioma cell lines (data not shown). Effective miR-1246 knockdown was reliably achieved in KNS42 cells, which were subjected to further functional experiments.

Infection of lentiviral-based miRNA plasmid miR-487b in the KNS42 cells led to $\sim 6 \times$ overexpression of miR-487b compared with control cells (Figures 5a and b). miR-487b overexpression led to a decrease in colony formation in soft agar $(30 \%)$ $(P<0.05)$ (Figure 5c) and decreased expression of the neural stem cell markers, known to be predicted targets of miR-487b, PROM1, and Nestin (but not WNT5A) (Figure 5d). miR-487b overexpression had no significant effect on cell growth, proliferation, sensitivity to temozolomide, migration, or invasion (Supplementary Figure 1). Inhibition of miR-1246 with the miR-1246 locker resulted in miR-1246 underexpression (Supplementary Figure 2B). There was no downregulation of predicted miR-1246 targets, or effect on cell growth, proliferation, sensitivity to temozolomide, migration, invasion, or colony formation in soft agar (Supplementary Figure 2).

\section{Discussion}

Alterations in microRNA levels have emerged as an important mechanism in cancer, including glial tumors. ${ }^{19,20,33,34}$ MicroRNA regulation also appears to have an important role in pediatric and low-grade glial neoplasms. For example, overexpression of
miR-21 and downregulation of miR-124 and miR-129 is common in pilocytic astrocytoma compared with non-neoplastic brain. ${ }^{22,23}$ These differences appear to be accentuated in neurofibromatosis type 1-associated pilocytic astrocytomas in particular, ${ }^{23}$ and were also present in our current study of a broader spectrum of pathologies.

Very little is known about differential microRNA expression in other low-grade pediatric and circumscribed gliomas and glioneuronal tumors. In a recent study, a set of microRNAs associated with inflammation (miR-146a, miR-21, and miR-155) were differentially expressed in ganglioglioma and peritumoral cortex. ${ }^{28}$ Another study demonstrated increased miR-146a levels in glioneuronal tumors, possibly as a negative feedback loop to an astrocytemediated inflammatory response. ${ }^{27}$

In our study, the directionality of altered microRNA expression of these low-grade gliomas was similar to each other and to microRNAs identified in previously published high-grade gliomas studies that were summarized in a recent meta-analysis (Figure 6). ${ }^{35}$ Many of these alterations are consistent in directionality with malignancies from other sites, such as miR-21a-5p, which is upregulated in many tumor types. ${ }^{23,36}$ One notable exception was miR-9, which was downregulated in subependymal giant cell astrocytomas but slightly upregulated in the other low-grade tumors, namely in pilocytic astrocytomas. This is one of the most commonly cited upregulated microRNAs in glioblastomas and is often upregulated in other malignant tumor types. ${ }^{35}$ One microRNA that was significantly upregulated in several low-grade gliomas and glioneuronal tumors was miR-34a, which is frequently downregulated in other malignant tumors and is thought to act as a tumor suppressor. ${ }^{37}$ In studies of glioblastoma, miR-34a regulation has been inconsistent, with some studies showing downregulation as compared with brain in the proneural subtype, ${ }^{38}$ but other studies showing upregulation in glioblastoma as compared with anaplastic astrocytoma. ${ }^{35,39}$ In our study, miR-34a was consistently upregulated in the glial and glioneuronal tumors as shown both in microarray results and in RT-PCR validation studies. Further study is necessary to determine the phenotype conferred by alterations in microRNAs, such as miR-9-5p and miR-34a, that have divergent expression in different glioma types and grades.

Regarding microRNA regulation by specific pathologic subtype, it was of interest that subependymal giant cell astrocytomas and rosette-forming glioneuronal tumors clustered together, farthest from nonneoplastic brain. These tumors share in common a predominant intraventricular location, and conceivably are less likely to have non-neoplastic brain contamination. However, they also are linked at the biologic level by having frequent alterations in the PI3K/mTOR signaling pathways. Subependymal giant cell astrocytoma is frequently associated with tuberous sclerosis, characterized at the genetic level 
miR-487b in glioma

a
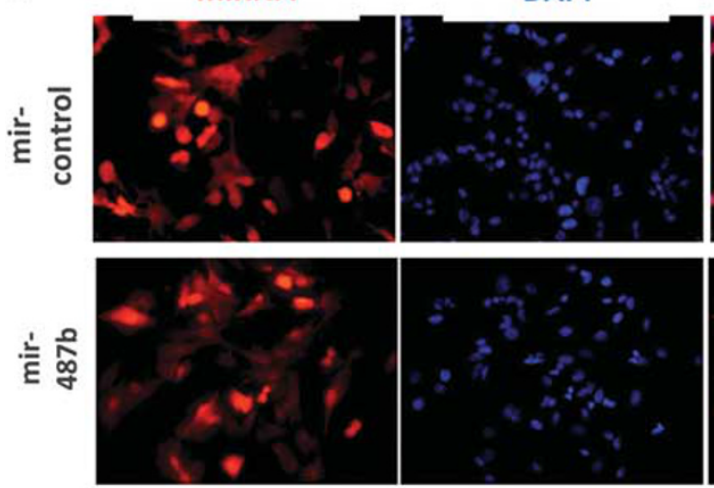

C

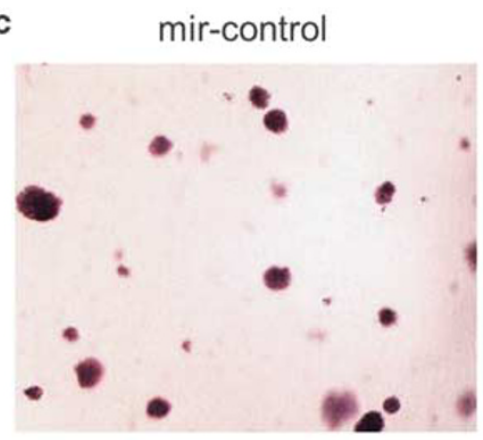

d

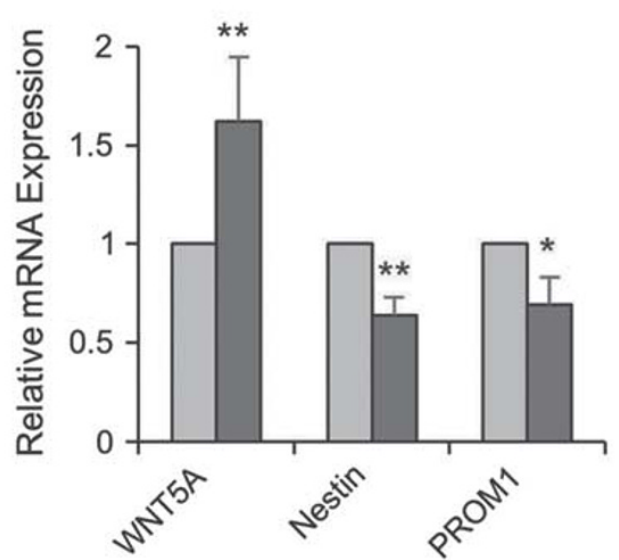

b
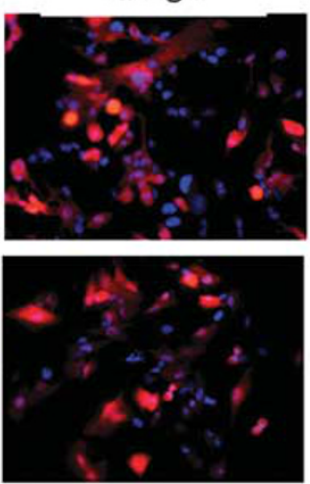

mir-487b

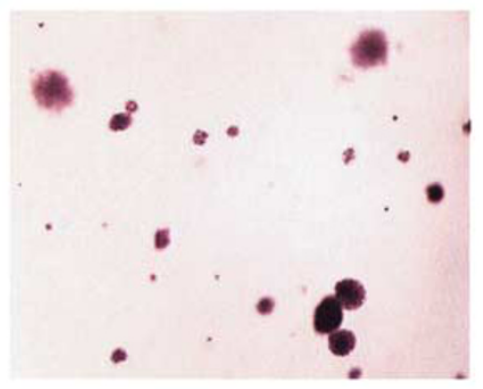

$\square$ mir-control

hsa-mir-487b

Figure 5 mir-487b overexpression in KNS42 cells affects expression of stem cell markers and colony formation in soft agar. (a) miRNA expression in virus infected KNS42 cells with mir-control or mir-487b (red), DAPI for nuclear staining (blue). (b) mi-487b overexpression in KNS42 cells infected with mir-487b. ${ }^{*} P<0.01$ compared with mir-control cells. (c) Soft agar clonogenic assay. Colony numbers of KNS42 cells infected with mir-control or mir-487b were counted after 21 days culture in 6-well plates. (d) qRT-PCR shows downregulation of Nestin and PROM1 and upregulation of WNT5A after mir-487b overexpression in KNS42 cells. All data were normalized to HPRT1. ${ }^{*} P<0.05,{ }^{*} P<0.01$.

by germline inactivation of the TSC1 or TSC2 tumor suppressor genes, whereas rosette-forming glioneuronal tumor has frequent mutations in PIK3CA. ${ }^{40}$ Activation of this pathway through TSC1 deletion in mouse and human cell lines has been shown to cause a global inhibition of microRNA biogenesis through the degradation of Drosha. ${ }^{41}$ Conversely, upregulation of the PTEN-inhibitor microRNA miR-21 has been shown to occur as a result of rapamycin inhibition, likely as a mechanism of negative feedback. ${ }^{42}$ This microRNA was frequently upregulated in the low-grade gliomas, including subependymal giant cell astrocytoma, as evaluated by both Nanostring hybridization screening and RTPCR validation.

In our study, we focused on two microRNAs for functional validation, miR-487b and miR-1246, as neither have previously been functionally validated as participating in gliomagenesis, and both have significant alterations in expression in low-grade glial and glioneuronal tumors by both Nanostring and RT-PCR assays. Although miR487b has been 


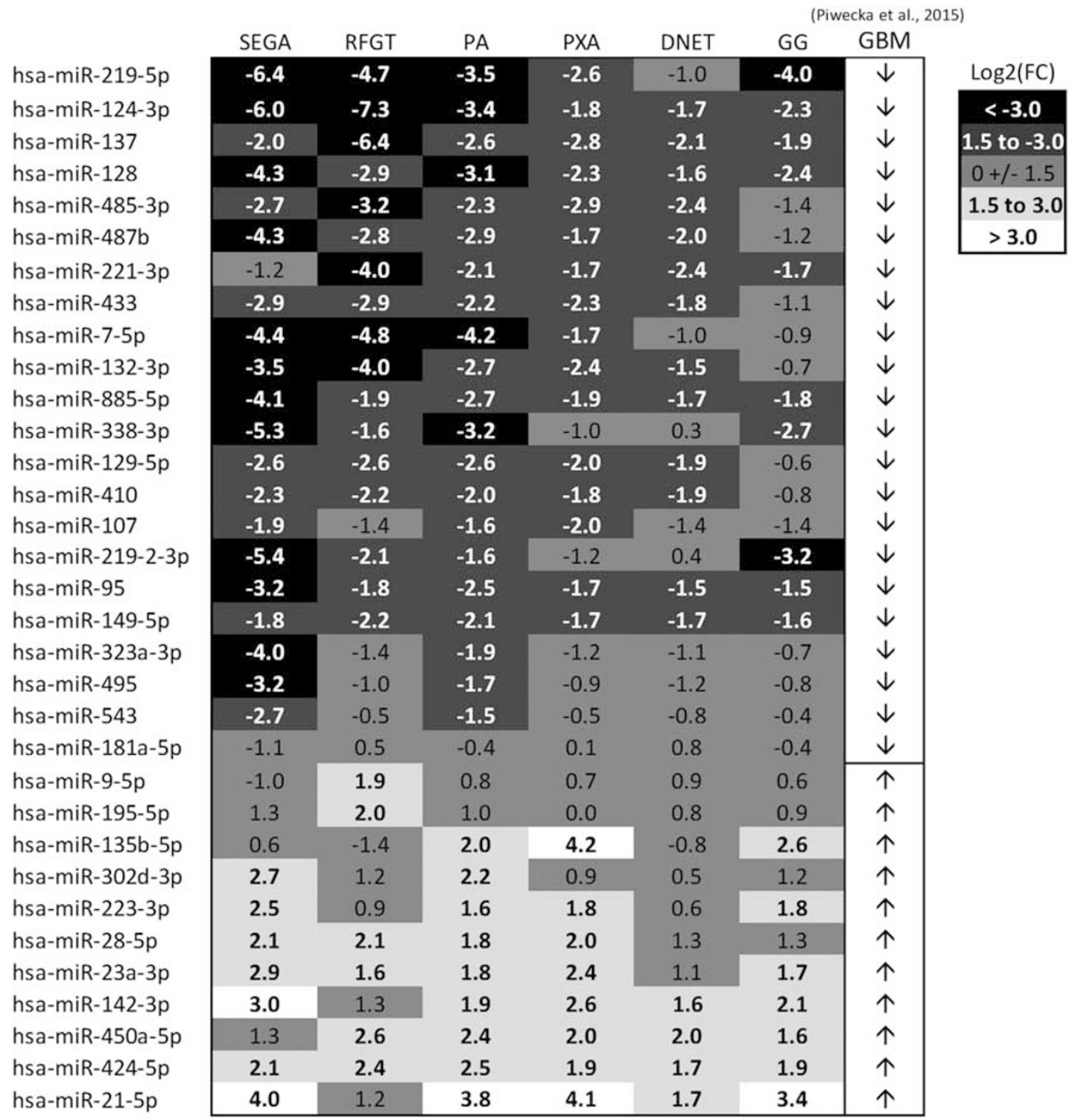

Figure 6 microRNA expression in lower grade gliomas compared with glioblastoma. Mean $\log _{2}$ (fold change) from normal brain for the tumor types with $n>3$ in our data set (subependymal giant cell astrocytoma, pilocytoic astrocytoma, pleomorphic xanthoastrocytoma, ganglioglioma, and dysembryoplastic neuroepithelial tumor)) shows similar directionality in expression to previous studies of glioblastoma (GBM) summarized in a recent meta-analysis. ${ }^{35}$

identified as downregulated in gliomas, its functional role in glial neoplasms has not been explored. In the current study, miR-487b overexpression led to decreased colony formation in soft agar and decreased levels of the neural stem cell markers nestin and PROM1 in a pediatric glioma cell line. The results of these functional experiments were intriguing, although they were performed on a pediatric high-grade glioma cell line (KNS42), rather than in the pediatric low-grade glioma cell lines that we had available (Res186, Res259). This approach was necessary for technical reasons, as KNS42 cells grow as neurospheres, therefore being more appropriate for the study of stem cell-like properties. In addition, KNS42 maintains high levels of miR-487b stem cell targets in culture (eg, PROM1, Nestin) and miR-1246 knockdown was successful in this cell line in our hands. As more appropriate models of pediatric low-grade glioma become available, similar experiments may be performed in the future to more accurately clarify the role of these microRNAs in pediatric low-grade glioma at the functional level.

miR-487 maps to chromosome locus 14q32.31, a region that is often deleted in high-risk neuroblastoma, and its loss is an indicator of poor prognosis in a manner that is independent of $M Y C$ expression. ${ }^{43,44}$ It is part of a parentally imprinted microRNA cluster that contains several other microRNAs that were frequently under-expressed in tumors in this study, including miR-485-3p, 
miR-410, miR-323a-3p, miR-495, and miR-543. Tested miR-487b targets in this context include components of neurodevelopmental and oncogenic signaling pathways WNT5a, BMI1, MYC, KRAS, and SUZ12 ${ }^{45}$ Interestingly, miR-487b may also be regulated by DNA methylation in tumorigenesis, as a response to cigarette smoke, a proposed mechanism for its involvement in lung tumors. ${ }^{45,46}$ The 14q32.31 cluster also appears to be differentially regulated in an epigenetic manner at different stages of stem cell reprogramming. ${ }^{47}$ miR-487b specifically appears to be relevant to CNS pathology, being upregulated in the blood of patients with ischemic stroke, and also promotes angiogenesis. ${ }^{48,49}$ Notably, it is downregulated in glioblastomas along with other microRNAs within the 14q32.31 cluster compared with non-neoplastic brain in some studies. ${ }^{50,51}$ As the targets of these genes are enriched in glial-specificity, this cluster may have an important role in glial tumor biology. ${ }^{50}$

In contrast to miR-487b, miR-1246 was consistently overexpressed in low-grade glioma and glioneuronal tumors in our study by microarray and RTPCR. miR-1246 maps to chromosome region 2q31.1. It is a target of the tumor suppressor p53, ${ }^{52-54}$ but has been found to be upregulated in various cancers. For instance, miR-1246 is upregulated in neuroblastoma cells, ${ }^{55}$ and serum exosomes of pancreatic and esophageal cancer patients; ${ }^{56,57}$ it promotes sphere formation and chemoresistance in pancreatic cancer, ${ }^{58}$ stemness and invasion in lung cancer, ${ }^{59}$ and enhances migration and invasion in hepatocellular carcinoma cell lines. ${ }^{60}$ This microRNA is also abundant in the exosomes of neural stem cells. ${ }^{61}$ One notable technical difficulty in studying miR-1246, however, is the inclusion of this sequence in the transcript for the small noncoding nuclear RNA RNAU2-1f, which has also been identified as a circulating RNA upregulated in tumorigenesis, that can be alternately spliced to produce miR-1246, and possibly miR-1290. ${ }^{62,63}$ Future sequencing studies may be necessary to determine the extent to which the RNA hybridization assays for miR-1246 are also, or exclusively, recognizing RNAU2-1f. If this is true in this case, RNAU2-1f may be a useful circulating biomarker for glioma growth. In addition, if miR-1246 does not function independently of RNAU2-1f in glial tumorigenesis, this may explain its lack of a tumorigenic role in cell culture experiments.

In summary, differential microRNA expression is a feature of a variety of low-grade, primarily pediatric, glioma, and glioneuronal tumor types. miR-487b appears to inhibit the expression of stem cell markers and properties in vitro, a finding that should be validated in the future in larger studies. These studies have a potential to increase our basic understanding of the genetic and epigenetics mechanisms responsible for gliomagenesis, and lead to the development of rational biomarkers and therapeutic targets.

\section{Acknowledgments}

This work was supported by the Childhood Brain Tumor Foundation (to FJR), the Pilocytic/Pilomyxoid Astrocytoma Fund including Lauren's First and Goal (to FJR) and Research to Prevent Blindness (MAV); samples quality assessment and microarray analysis were conducted at The Sidney Kimmel Cancer Center Microarray Core Facility at Johns Hopkins University, supported by National Institutes of Health (P30 CA006973) entitled Regional Oncology Research Center.

\section{Disclosure/conflict of interest}

The authors declare no conflict of interest.

\section{References}

1 Bar EE, Lin A, Tihan $\mathrm{T}$, et al. Frequent gains at chromosome $7 q 34$ involving BRAF in pilocytic astrocytoma. J Neuropathol Exp Neurol 2008;67: 878-887.

2 Forshew T, Tatevossian RG, Lawson AR, et al. Activation of the ERK/MAPK pathway: a signature genetic defect in posterior fossa pilocytic astrocytomas. J Pathol 2009;218:172-181.

3 Jones DT, Kocialkowski S, Liu L, et al. Tandem duplication producing a novel oncogenic BRAF fusion gene defines the majority of pilocytic astrocytomas. Cancer Res 2008;68:8673-8677.

4 Pfister S, Janzarik WG, Remke M, et al. BRAF gene duplication constitutes a mechanism of MAPK pathway activation in low-grade astrocytomas. J Clin Invest 2008;118:1739-1749.

5 Sievert AJ, Jackson EM, Gai X, et al. Duplication of 7q34 in pediatric low-grade astrocytomas detected by high-density single-nucleotide polymorphism-based genotype arrays results in a novel BRAF fusion gene. Brain Pathol 2009;19:449-458.

6 Jones DT, Hutter B, Jager N, et al. Recurrent somatic alterations of FGFR1 and NTRK2 in pilocytic astrocytoma. Nat Genet 2013;45:927-932.

7 Hutt-Cabezas M, Karajannis MA, Zagzag D, et al. Activation of mTORC1/mTORC2 signaling in pediatric low-grade glioma and pilocytic astrocytoma reveals mTOR as a therapeutic target. Neuro Oncol 2013;15: 1604-1614.

8 Kaul A, Chen YH, Emnett RJ, et al. Pediatric gliomaassociated KIAA1549:BRAF expression regulates neuroglial cell growth in a cell type-specific and mTOR-dependent manner. Genes Dev 2012;26: 2561-2566.

9 Ramkissoon LA, Horowitz PM, Craig JM, et al. Genomic analysis of diffuse pediatric low-grade gliomas identifies recurrent oncogenic truncating rearrangements in the transcription factor MYBL1. Proc Natl Acad Sci U S A 2013;110:8188-8193.

10 Zhang J, Wu G, Miller CP, et al. Whole-genome sequencing identifies genetic alterations in pediatric low-grade gliomas. Nat Genet 2013;45:602-612.

11 Chan JA, Krichevsky AM, Kosik KS. MicroRNA-21 is an antiapoptotic factor in human glioblastoma cells. Cancer Res 2005;65:6029-6033. 
12 Ciafre SA, Galardi S, Mangiola A, et al. Extensive modulation of a set of microRNAs in primary glioblastoma. Biochem Biophys Res Commun 2005;334: 1351-1358.

13 Kefas B, Godlewski J, Comeau L, et al. microRNA-7 inhibits the epidermal growth factor receptor and the Akt pathway and is down-regulated in glioblastoma. Cancer Res 2008;68:3566-3572.

14 Medina R, Zaidi SK, Liu CG, et al. MicroRNAs 221 and 222 bypass quiescence and compromise cell survival. Cancer Res 2008;68:2773-2780.

15 Shi L, Cheng Z, Zhang J, et al. hsa-mir-181a and hsa-mir-181b function as tumor suppressors in human glioma cells. Brain Res 2008;1236:185-193.

16 Sumazin P, Yang X, Chiu HS, et al. An extensive microRNA-mediated network of RNA-RNA interactions regulates established oncogenic pathways in glioblastoma. Cell 2011;147:370-381.

17 Li D, Chen P, Li XY, et al. Grade-specific expression profiles of miRNAs/mRNAs and docking study in human grade I-III astrocytomas. Omics 2011;15: 673-682.

18 Nicoloso MS, Calin GA. MicroRNA involvement in brain tumors: from bench to bedside. Brain Pathol 2008;18:122-129.

19 Huse JT, Brennan C, Hambardzumyan D, et al. The PTEN-regulating microRNA miR-26a is amplified in high-grade glioma and facilitates gliomagenesis in vivo. Genes Dev 2009;23:1327-1337.

$20 \mathrm{Lu}$ J, Getz G, Miska EA, et al. MicroRNA expression profiles classify human cancers. Nature 2005;435: 834-838.

21 White FV, Anthony DC, Yunis EJ, et al. Nonrandom chromosomal gains in pilocytic astrocytomas of childhood. Hum Pathol 1995;26:979-986.

22 Birks DK, Barton VN, Donson AM, et al. Survey of MicroRNA expression in pediatric brain tumors. Pediatr Blood Cancer 2011;56:211-216.

23 Ho CY, Bar E, Giannini C, et al. MicroRNA profiling in pediatric pilocytic astrocytoma reveals biologically relevant targets, including PBX3, NFIB, and METAP2. Neuro Oncol 2013;15:69-82.

24 Sredni ST, Huang CC, Bonaldo Mde F, et al. MicroRNA expression profiling for molecular classification of pediatric brain tumors. Pediatr Blood Cancer 2011;57: 183-184.

25 Jones TA, Jeyapalan JN, Forshew T, et al. Molecular analysis of pediatric brain tumors identifies microRNAs in pilocytic astrocytomas that target the MAPK and NF-kappaB pathways. Acta Neuropathol Commun 2015;3:86.

26 Costa FF, Bischof JM, Vanin EF, et al. Identification of microRNAs as potential prognostic markers in ependymoma. PLoS One 2011;6:e25114.

27 Iyer A, Zurolo E, Prabowo A, et al. MicroRNA-146a: a key regulator of astrocyte-mediated inflammatory response. PLoS One 2012;7:e44789.

28 Prabowo AS, van Scheppingen J, Iyer AM, et al. Differential expression and clinical significance of three inflammation-related microRNAs in gangliogliomas. J Neuroinflamm 2015;12:97.

29 Bax DA, Little SE, Gaspar N, et al. Molecular and phenotypic characterisation of paediatric glioma cell lines as models for preclinical drug development. PLoS One 2009;4:e5209.

30 Hanaford AR, Archer TC, Price A, et al. DiSCoVERing innovative therapies for rare tumors: combining genetically accurate disease models with in silico analysis to identify novel therapeutic targets. Clin Cancer Res 2016;22:3903-3914.

31 Lewis BP, Burge CB, Bartel DP. Conserved seed pairing, often flanked by adenosines, indicates that thousands of human genes are microRNA targets. Cell 2005;120: 15-20.

32 Busk PK. A tool for design of primers for microRNAspecific quantitative RT-qPCR. BMC Bioinformatics 2014;15:29.

33 Lai NS, Dong QS, Ding H, et al. MicroRNA-210 overexpression predicts poorer prognosis in glioma patients. J Clin Neurosci 2014;21:755-760.

34 Mucaj V, Lee SS, Skuli N, et al. MicroRNA-124 expression counteracts pro-survival stress responses in glioblastoma. Oncogene 2015;34:2204-2214.

35 Piwecka M, Rolle K, Belter A, et al. Comprehensive analysis of microRNA expression profile in malignant glioma tissues. Mol Oncol 2015;9:1324-1340.

36 Haider BA, Baras AS, McCall MN, et al. A critical evaluation of microRNA biomarkers in non-neoplastic disease. PLoS ONE 2014;9:e89565.

37 Hayes J, Peruzzi PP, Lawler S. MicroRNAs in cancer: biomarkers, functions and therapy. Trends Mol Med 2014;20:460-469.

38 Silber J, Jacobsen A, Ozawa T, et al. miR-34a repression in proneural malignant gliomas upregulates expression of its target PDGFRA and promotes tumorigenesis. PLoS One 2012;7:e33844.

39 Rao SAM, Santosh V, Somasundaram K. Genome-wide expression profiling identifies deregulated miRNAs in malignant astrocytoma. Mod Pathol 2010;23: 1404-1417.

40 Ellezam B, Theeler BJ, Luthra R, et al. Recurrent PIK3CA mutations in rosette-forming glioneuronal tumor. Acta Neuropathol 2012;123:285-287.

41 Ye P, Liu Y, Chen C, et al. An mTORC1-Mdm2-Drosha axis for miRNA biogenesis in response to glucose- and amino acid-deprivation. Mol Cell 2015;57:708-720.

42 Trindade AJ, Medvetz DA, Neuman NA, et al. MicroRNA-21 is induced by rapamycin in a model of tuberous sclerosis (TSC) and lymphangioleiomyomatosis (LAM). PLoS One 2013;8:e60014.

43 Gattolliat CH, Thomas L, Ciafre SA, et al. Expression of miR-487b and miR-410 encoded by $14 \mathrm{q} 32.31$ locus is a prognostic marker in neuroblastoma. $\mathrm{Br} \mathrm{J}$ Cancer 2011;105:1352-1361.

44 Gattolliat CH, Le Teuff G, Combaret V, et al. Expression of two parental imprinted miRNAs improves the risk stratification of neuroblastoma patients. Cancer Med 2014;3:998-1009.

$45 \mathrm{Xi} \mathrm{S}, \mathrm{Xu} \mathrm{H}$, Shan J, et al. Cigarette smoke mediates epigenetic repression of miR-487b during pulmonary carcinogenesis. J Clin Invest 2013;123:1241-1261.

46 Stewart DJ. Wnt signaling pathway in non-small cell lung cancer. J Natl Cancer Inst 2014;106:djt356.

47 Clancy JL, Patel HR, Hussein SM, et al. Small RNA changes en route to distinct cellular states of induced pluripotency. Nat Commun 2014;5:5522.

48 Jickling GC, Ander BP, Zhan X, et al. microRNA expression in peripheral blood cells following acute ischemic stroke and their predicted gene targets. PLoS One 2014;9:e99283.

49 Feng N, Wang Z, Zhang Z, et al. miR-487b promotes human umbilical vein endothelial cell proliferation, migration, invasion and tube formation through regulating THBS1. Neurosci Lett 2015;591:1-7. 
50 Laddha SV, Nayak S, Paul D, et al. Genomewide analysis reveals downregulation of miR-379/ miR-656 cluster in human cancers. Biol Direct 2013;8: 10.

51 Skalsky RL, Cullen BR. Reduced expression of brainenriched microRNAs in glioblastomas permits targeted regulation of a cell death gene. PLoS One 2011;6: e24248.

52 Liao JM, Zhou X, Zhang Y, et al. MiR-1246: a new link of the p53 family with cancer and Down syndrome. Cell Cycle 2012;11:2624-2630.

53 Zhang Y, Liao JM, Zeng SX, et al. p53 downregulates Down syndrome-associated DYRK1A through miR-1246. EMBO Rep 2011;12:811-817.

54 Zhang Q, Cao LY, Cheng SJ, et al. p53-induced microRNA-1246 inhibits the cell growth of human hepatocellular carcinoma cells by targeting NFIB. Oncol Rep 2015;33:1335-1341.

$55 \mathrm{Xu}$ LJ, Jiang $\mathrm{T}$, Zhao $\mathrm{W}$, et al. Parallel mRNA and microRNA profiling of HEV71-infected human neuroblastoma cells reveal the up-regulation of miR-1246 in association with DLG3 repression. PLoS One 2014;9: e95272.

56 Madhavan B, Yue S, Galli U, et al. Combined evaluation of a panel of protein and miRNA serum-exosome biomarkers for pancreatic cancer diagnosis increases sensitivity and specificity. Int J Cancer 2015;136: 2616-2627.
57 Takeshita N, Hoshino I, Mori M, et al. Serum microRNA expression profile: miR-1246 as a novel diagnostic and prognostic biomarker for oesophageal squamous cell carcinoma. Br J Cancer 2013;108:644-652.

58 Hasegawa S, Eguchi $\mathrm{H}$, Nagano $\mathrm{H}$, et al. MicroRNA-1246 expression associated with CCNG2mediated chemoresistance and stemness in pancreatic cancer. Br J Cancer 2014;111:1572-1580.

59 Kim G, An HJ, Lee MJ, et al. Hsa-miR-1246 and hsamiR-1290 are associated with stemness and invasiveness of non-small cell lung cancer. Lung Cancer 2016;91:15-22.

60 Sun Z, Meng C, Wang S, et al. MicroRNA-1246 enhances migration and invasion through CADM1 in hepatocellular carcinoma. BMC Cancer 2014;14:616.

61 Stevanato L, Thanabalasundaram L, Vysokov N, et al. Investigation of content, stoichiometry and transfer of miRNA from human neural stem cell line derived exosomes. PLoS One 2016;11:e0146353.

62 Mazieres J, Catherinne C, Delfour O, et al. Alternative processing of the U2 small nuclear RNA produces a 1922nt fragment with relevance for the detection of nonsmall cell lung cancer in human serum. PLoS One 2013;8:e60134.

63 Baraniskin A, Zaslavska E, Nöpel-Dünnebacke S, et al. Circulating U2 small nuclear RNA fragments as a novel diagnostic biomarker for primary central nervous system lymphoma. Neuro Oncol 2016;18:361-367.

Supplementary Information accompanies the paper on Modern Pathology website (http://www.nature.com/ modpathol) 